\title{
Classically conditioned modulation of pain depends on stimulus intensity
}

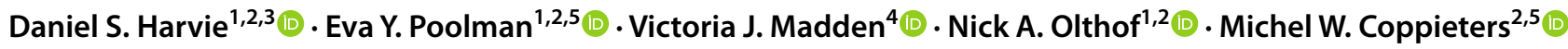

Received: 5 August 2021 / Accepted: 1 December 2021 / Published online: 11 February 2022

(c) The Author(s) 2022

\begin{abstract}
Innocuous cues that become associated with pain can enhance pain. This is termed classically conditioned hyperalgesia. The size of this effect varies under different conditions. We aimed to test whether the sensitising effect of pain-associated cues depends on the intensity of the paired test stimulus. To do this, two virtual reality environments were paired with either painful or non-painful vibrotactile stimuli in a counterbalanced fashion. The differential effect of the two environments was evaluated using pain intensity ratings of paired electrocutaneous test stimuli at three different intensity levels. Forty healthy participants were included in the study; 30 participants experienced sufficient pain during the learning phase and were included in the main analysis. An effect of environment $(p=0.014)$ and interaction between environment and test stimulus intensity was found $(p=0.046)$. Only the most intense test stimulus was modulated by environment. While the effect was small, the results are consistent with the proposition that pain-associated cues may induce hyperalgesia to some degree, under certain conditions. In particular, results highlight the potential relevance of stimulus intensity during and after the initial painful experience. Further attention is needed to comprehensively understand the variables that impact classically conditioned hyperalgesia.
\end{abstract}

Keywords Classical conditioning $\cdot$ Chronic pain $\cdot$ Psychophysics $\cdot$ Associative learning $\cdot$ Conditioned hyperalgesia Nocebo

Communicated by Bill J Yates.

Michel W. Coppieters

m.coppieters@griffith.edu.au

1 School of Health Sciences and Social Work, Griffith University, Brisbane and Gold Coast, Australia

2 Menzies Health Institute Queensland, Griffith University, 170 Kessels Road, Nathan, Brisbane and Gold Coast, QLD 4111, Australia

3 IIMPACT, Allied Health and Human Performance, University of South Australia, Cape Town, South Africa

4 Pain Unit, Department of Anaesthesia and Perioperative Medicine, Neuroscience Institute, University of Cape Town, Cape Town, South Africa

5 Amsterdam Movement Sciences, Faculty of Behavioural and Movement Sciences, Vrije Universiteit Amsterdam, Van der Boechorststraat 9, 1081 BT Amsterdam, The Netherlands

\section{Introduction}

Classical conditioning is the process whereby a normally neutral stimulus can develop the capacity to evoke behavioural responses by becoming associated with innately meaningful stimuli (Pavlov 1928). In pain research, this paradigm has been used to understand pain-related fear and its connection to fear of movement (Vlaeyen et al. 1995; Vlaeyen and Linton 2000, 2012). Here, fear is viewed as an innate response to pain, such that, after formation of an association between movement and pain, fear becomes a learned response to movement (Lethem et al. 1983; Hamm et al. 1989; Van Damme et al. 2004; Liu 2011; Meulders et al. 2011; Glotzbach et al. 2012; Moseley and Vlaeyen 2015). The Imprecision Hypothesis proposes a pain learning process that may occur in parallel to any learning of pain-related fear (Moseley and Vlaeyen 2015). Here, pain is viewed as an innate response to nociception, such that, after formation of an association between movement and painful nociceptive events, pain becomes a learned response to movement. Moreover, it proposes that other cues, such as environmental 
contexts or tactile cues associated with nociceptive events, could also contribute to pain.

No laboratory evidence exists that pain can be evoked by an isolated pain-associated cue, although there is evidence that a pain-associated cue can increase pain intensity and lower pain thresholds (Madden et al. 2016). Current data suggest that stimuli associated with nociception can increase pain intensity by 7.4 on a 0 to 100 pain rating scale (Madden et al. 2016). While changes in pain of less than $2 / 10$ or $30 \%$ are not considered meaningful in clinical scenarios (Farrar et al. 2001), we hypothesise that effects may be restrained by low ecological validity and other deficits in laboratory methods. Indeed, there is already evidence of variables that may impact the strength of the effect. For example, the magnitude of the effect is predicted by the intensity of painful stimulation during the learning phase (Jensen et al. 2012, 2015; Harvie et al. 2016), likely because greater intensity invokes a stronger 'learning signal'.

Further potential influencers of classically conditioned pain modulation may be derived from models of perception and well-established learning principles. In the Bayesian framework of perception, perceptions emerge from a best estimate of reality, based on the integration of relevant information from various sources (Knill and Pouget 2004; Meyniel et al. 2015). Here, a signal's influence on perception (i.e. its relative weight) depends on factors such as its salience relative to other relevant signals. This would predict that a pain-associated cue may have more influence on perceived pain when paired with a painful stimulus of lower intensity, since the pain-associated cue would have more relative weight. Another possible influence is stimulus belongingness, which expresses the principle that certain stimuli may be more likely to become associated because of their functional relevance (Domjan and Galef 1983).

In the current study, we aimed to investigate potential modifiers of the pain-enhancing effect of pain-associated cues. Based on the Bayesian view of perception, we hypothesised that pain-associated Virtual Reality (VR) environments would have their greatest pain-enhancing effect on painful stimuli of lower intensity.

\section{Methods}

\section{Participants}

Participants were recruited through advertisements on a university campus. Participants were not informed of the specific research question or the study hypotheses, but were informed that we were using VR to better understand pain. Participants were eligible if they were healthy, pain-free, and over the age of 18 years. They were excluded if they had a history of chronic ( $>3$ months) pain, a diagnosed neurological or psychiatric disease, were taking analgesics or psychoactive drugs, were pregnant, or had an electronic implant.

For each participant, the age, sex and questionnaires to assess depression [PHQ-9: Patient Health Questionnaire-9 (Kroenke and Spitzer 2002)], general anxiety [GAD-7: General Anxiety Disoreder-7 (Lowe et al. 2008)] and pain beliefs [FPQ-9: Fear of Pain Questionnaire-9 (McNeil et al. 2018) and PCS: Pain Catastrophizing Scale (Sullivan et al. 1995)] were collected prior to the experiment.

\section{Experiment design overview}

In classical conditioning studies, a learning phase pairing a neutral stimulus with an innately response-evoking stimulus is used, in order that the neutral stimulus itself becomes response-evoking - by virtue of its new association with the other stimulus. Following this, a test phase is used to measure the degree to which the initially neutral stimulus has acquired the expected response-evoking properties.

In this study, the learning phase (Fig. 1.II) involved pairing two initially neutral VR environments with either painful nociceptive stimuli or non-painful vibrotactile stimuli. The test phase (Fig. 1.III) involved assessing the degree to which the pain-associated VR environment modulated pain relative to the vibrotactile-associated VR environment. The specific environment paired with each stimulus was counterbalanced among participants. During the learning phase, each environment was presented 10 times, for $25 \mathrm{~s}$, in a randomised order. In the test phase, the effect of each context on pain sensitivity was tested by presenting electrocutaneous test stimuli in each environment, and asking participants to rate perceived pain intensity.

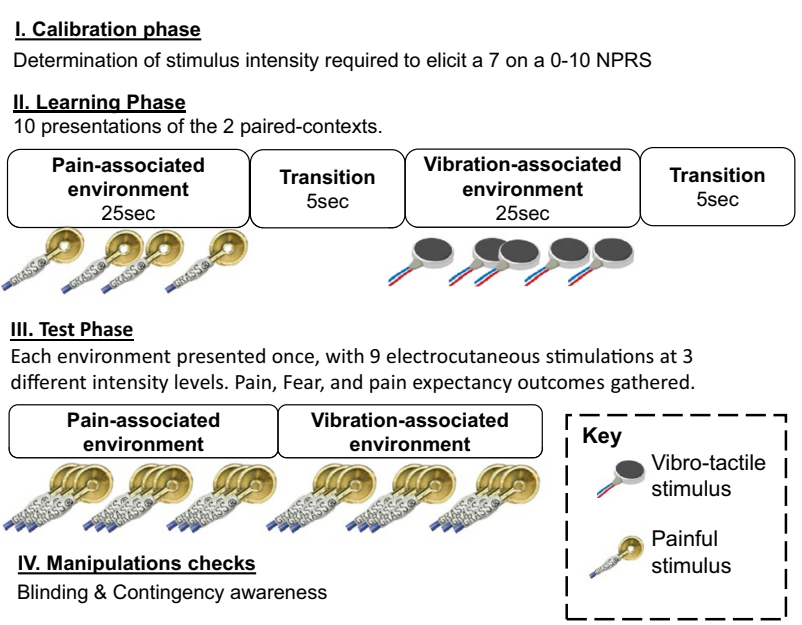

Fig. 1 Stages of the experiment 


\section{Stimulus equipment}

\section{Electrocutaneous stimulation}

Electrocutaneous stimuli were used to deliver painful stimulations. Electrocutaneous stimuli were generated using a Digitimer D185 MultiPulse stimulator (Digitimer Ltd, Welwyn Garden City, Hertfordshire, UK) with $50 \mu$ s pulse duration, with a maximum current output of $1.5 \mathrm{~A}$ and an inter-pulse interval of $9.9 \mathrm{~ms}$. The stimuli were delivered via Genuine Grass Brand 10 mm gold-plated cup electrodes. The intensity level was varied by altering the number of pulses contained within each individual stimulus. The maximum intensity was a stimulus of approximately $90 \mathrm{~ms}$ duration, made up of a train of 9 pulses. The voltage of the stimulations was individually calibrated such that the 9-pulse stimulus would evoke a pain response rated as 7 out of 10 on the Numerical Pain Rating Scale (NPRS); where $0=$ no sensation, $3=$ first instance of pain, $5=$ moderate pain, $7=$ significant pain, and $10=$ intolerable pain. A staircase method of calibration, with increasing and decreasing intensities (ranging between 10 and $50 \mathrm{~V}$ ), was performed on the dorsum of the left foot until NPRS 7 was reached three times. These parameters were then applied to the right foot to verify that this was also a NPRS of 7 on the opposite side. Notably, the left foot stimulations were used simply to increase experimental unpredictability, and only pain ratings for the right foot were used in the analysis.

\section{Vibrotactile stimulation}

Non-nociceptive vibrotactile stimuli were used as the control stimulus. This consisted of a $10 \mathrm{~mm}, 3 \mathrm{~V}$ vibration motor, oscillating at $200 \mathrm{~Hz}$ for approximately $500 \mathrm{~ms}$ duration. The motors were placed $1 \mathrm{~cm}$ medial to the electrocutaneous stimulus on each foot.

\section{Environments as conditioning stimuli}

VR was used to present different environmental contexts. These were presented using custom VR software (MoOViWearable Computer Lab) on a Windows PC (Alienware 17 R4, P31E, China). Three 'neutral' environments were sourced from databases of equirectangular $\left(360^{\circ}\right)$ photographic images. One indoor and one forest scene functioned as the contextual conditioned stimuli (see Supplementary File 1). Based on previous research that showed VR environments do not differentially influence pain, the environments were regarded as neutral with respect to pain at baseline (Smith et al. 2017).

\section{Procedure}

During the experiment, participants were seated in a chair, VR headset in place, and stimulators on both feet. Pain ratings were collected for all stimuli during the third, fifth and eighth environment presentations during the learning phase, so that we could assess if stimuli were sufficiently painful during learning. Calibration was labelled as successful if at least half $(5 / 10)$ of these stimuli were rated as equal or above NPRS 5 ('moderate' pain).

During the test phase, each environment was presented once (Fig. 1.III) in counterbalanced order among participants. Nine electrocutaneous stimuli, with a 10-s inter-stimulus interval, were delivered in each test phase environment. These stimuli were presented at three different intensities (low (1 pulse), medium ( 3 pulses) and high (5 pulses)) and in block randomised order.

\section{Expectancy and fear learning}

Expectancy and fear ratings were collected to explore the potential role of expectancy and fear in mediating pain modulation. These ratings were collected at the beginning of each test-phase environment prior to electrocutaneous stimulation. Questions were asked prior to electrocutaneous or vibrotactile stimulation via a digital audio recording. Participants rated the extent to which they expected to receive a painful stimulus on a 0 to 10 US expectancy scale, where $0=\mathrm{I}$ do not expect pain at all, and $10=\mathrm{I}$ fully expect to receive painful stimuli. Fear ratings were collected by asking participants how fearful they felt on a scale from 0 to 10 where $0=$ not at all fearful, and $10=$ extremely fearful.

\section{Blinding and contingency awareness}

Previous literature suggests that classical conditioning relates strongly to, and may depend on, propositional learning (Lovibond and Shanks 2002; Mitchell et al. 2009). Therefore, participants were asked after the experiment, " $A t$ any time during the experiment, were you able to tell when you were likely to get a painful electrical stimulus?' If yes, when did you receive them?" (Fig. 1.IV). Participants were labelled as contingency aware if they correctly identified which environment had been associated with pain during the learning phase. Additionally, although participants were naïve to the specific experimental aims at the beginning of the study, we assessed the persistence of blinding after the experiment by asking participants 'What do you think we are aiming to test in this study?'. 


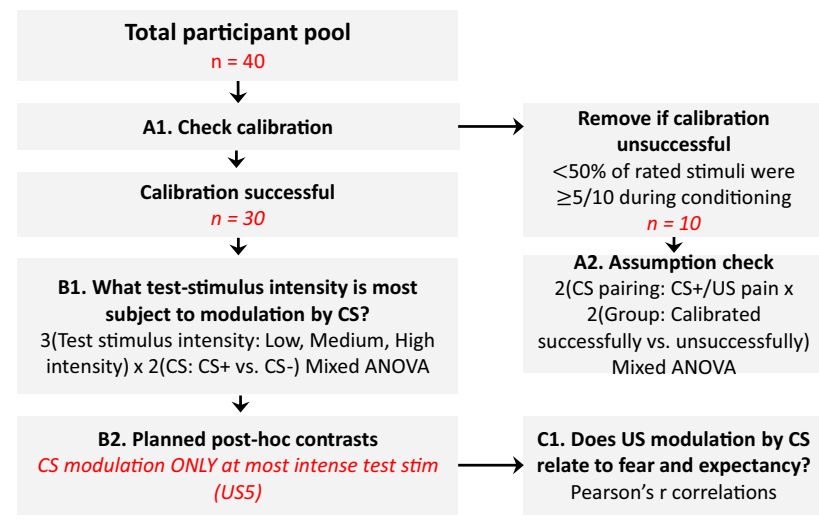

Fig. 2 Overview of the study and analysis procedure

\section{Data analysis and statistics}

For analysis and discussion, the abbreviation CS + (i.e. Conditioned Stimulus) was used to denote the pain-associated VR environment. The abbreviation CS- was used to denote the vibrotactile-associated VR environment. The abbreviation US (i.e. Unconditioned Stimulus) was used to denote the painful stimulus. The analysis plan is represented in Fig. 2, italicised text represents the flow of participants and data.

\section{Calibration check (Fig. 2, A1)}

Because prior studies have identified pain intensity as an important factor in achieving conditioned hyperalgesia, we planned to exclude cases where calibration did not result in sufficient pain (as defined above) during learning. To confirm that this a priori plan was appropriate, we analysed whether those with unsuccessful calibration $(n=10)$ failed to show classically conditioned hyperalgesia as expected (Fig. 2, A2). As planned, only participants reporting adequate pain intensity during the learning phase $(n=30)$ were used in further analyses. This also fit with the aim of the study to explore factors associated with a larger effect, and their potential to contribute to cumulative effect that is more meaningful.

\section{Primary analysis (Fig. 2, B1)}

For the primary analysis, we wanted to clarify which test stimulus intensity was most subject to modulation by the CS. For this, we used the test phase data in a 2 (CS: CS + vs. CS - ) $\times 3$ (Test stimulus intensity: Low, Medium, High intensity) repeated-measures ANOVA. We planned to then use contrasts to further probe differential classically conditioned effects at the different intensities (Fig. 2, B2). A differential effect of the VR environments was only shown for the high-intensity test stimulus.

\section{Secondary analysis}

To interrogate the potential relationship between pain and fear, and pain and expectancy, we used two Pearson's $r$ zeroorder correlations (Fig. 2, C1). Difference scores calculated by subtracting the scores reported in the pain-associated environment from those in the vibrotactile-associated environment were used for each bivariate correlation.

\section{Assumption checks}

Prior to analysis, data were checked for normality. Normality was tested using the Shapiro-Wilk test, and the eyeball test and skewness and kurtosis. Additionally, Mauchly's test of sphericity was used to see if the variances of the differences between all combinations of related groups were equal. Where the sphericity assumption was violated, the Huynh-Feldt correction was used. Tests were performed as two-tailed and the cut-off level for significance was set at $p<0.05$ for ANOVA testing. Bonferroni corrected $p$ values were employed for pairwise comparisons.

\section{Reporting}

Results were expressed in terms of absolute mean difference between pain during the pain-associated and vibration-associated contexts, as the mean difference expressed as a percentage (relative to the mean of all $\mathrm{CS}+$ and $\mathrm{CS}-$ ratings). Statistical significance, and the effect sizes Cohen's $d$ and partial eta-squared $\left(\eta_{p}^{2}\right)$ were also used where appropriate.

\section{Sample size}

Our sample size calculation was based on the primary within-subject analysis examining whether the three different test stimulus intensities would be differently modulated by the paired contexts. Although we did not have prior data to inform the calculation, we deemed it important to be able to detect at least a medium effect (e.g. $\left.\eta_{p}^{2}=0.06\right)$ with $80 \%$ power and alpha set at 0.05 . Based on these inputs, we estimated the need for at least 28 participants. Based on previous experiences (Harvie et al. 2016), substantial numbers of participants can report insufficient pain during the experiment despite calibration. Since we planned a priori to exclude such participants, we increased the sample size to 40 . 


\section{Results}

\section{Participants}

Forty healthy, pain-free volunteers [20 females, mean (SD) age $=29$ (7) years] met the inclusion criteria and participated in the study. There were no dropouts. Overall, participants were within normative ranges with respect to psychological scales including the PHQ-9 [3.2 (3.9)= no significant depressive symptoms], GAD-7 [3.5 (3.3)= minimal anxiety], FPQ-9 [21.5 $(6.5)=$ low levels of fear of pain], Pain Catastrophizing Scale $[12.3(7.8)=$ no clinically relevant pain catastrophizing].

\section{Calibration assessment}

Of the 40 participants, 10 were classified as having unsuccessful calibration due to experiencing insufficient pain. The 'Assumption check' ANOVA found no main effect of CS type $\left(F(1,38)=0.1, p=0.74, \eta_{p}^{2}=0.00\right)$ indicating no significant overall difference in pain ratings between the pain-associated context and the vibration-associated context (Fig. 3). However, there was a significant two-way interaction between calibration success and CS $(F(1,38)=5.7$, $p=0.022, \eta_{p}^{2}=0.189$ ). Paired $t$ tests (with a Bonferroni corrected significance threshold of $p=0.025)$ revealed a significant difference between CSs for the successfully calibrated group only $(t(29)=2.6, p=0.015, d=0.22)$ with a group mean (SD) of 7.3 (17.4)\% higher pain ratings in the painassociated context [mean (SD) NPRS $=3.5(1.0)]$ than in the vibration-associated context [3.2 (0.9)] (see Fig. 3). In the unsuccessfully calibrated group, there was no significant effect of CS $(t(9)=-1.5, p=0.176)$. Those who were

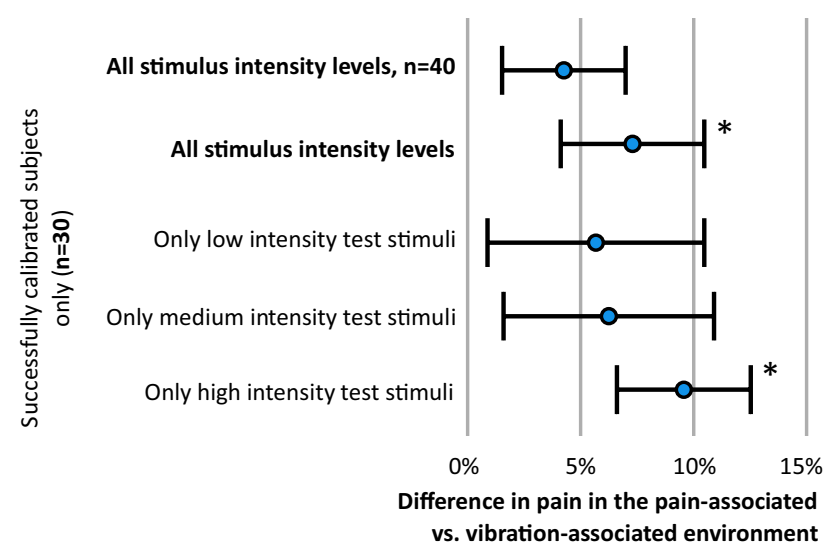

Fig. 3 The average percentage difference between CS +/US and CSUS pairings among participants. An asterisk is shown where a significant difference was found between pain- and vibration-associated environments classified as 'successfully calibrated' reported, on average, a pain intensity of $5.92(0.66) / 10$ during the learning phase. Those who were not classified as successfully calibrated reported, on average, a pain intensity of $3.73(0.98) / 10$. As planned, the main analyses proceeded using the 30 participants with 'successful' calibration.

\section{Primary analysis: which test stimulus intensity is most subject to modulation by the CS?}

Among those classified as reaching the 'successful' calibration threshold, the low-, medium-, and high-intensity test stimuli were rated as [mean $(\mathrm{SD})=1.8(0.9) / 10,3.8$ (1.12)/10 and $4.6(1.2) / 10$, respectively]. The primary analysis revealed a main effect of $\operatorname{CS}\left(F(1,29)=6.77, p=0.014, \eta_{p}^{2}\right.$ $=0.189)$ and a two-way interaction between CS and test stimulus intensity $\left(F(2,58)=3.3, p=0.046, \eta_{p}^{2}=101\right)$. Planned contrasts (with a Bonferroni corrected significance threshold of $p=0.017$ ) revealed a differential classically conditioned effect for the high-intensity test stimuli $(t(29)=3.37$, $p=0.002, d=0.34)$ corresponding to an $11.4(25.7) \%$ greater differential effect (mean diff: $0.40(0.66) / 10)$ in the painassociated (CS +) context [mean (SD): $4.76(1.20) / 10]$ than in the vibration-associated (CS -) context [mean (SD): 4.36 (1.14)/10] (see Fig. 3). There was no differential classically conditioned effect for medium intensity stimuli $(p=0.174)$ or low intensity test stimuli $(p=0.529)$.

Following the experimental procedure, 25 participants did not correctly guess the aim of the study and were classified as blinded, and all but seven participants correctly reported the experimental contingencies. The effect of stimulus blinding and contingency awareness on the main finding was examined using a 2 (Blinding: Blind vs. Unblinded) $\times 2$ (CS: CS + vs. CS -) and a 2 (Contingency: Aware vs. Unaware $) \times(2(\mathrm{CS}: \mathrm{CS}+$ vs. $\mathrm{CS}-)$ mixed ANOVA. There was no interaction with either contingency awareness or blinding (all $p>0.17$ ).

\section{Secondary analyses: relationship between the differential classically conditioned effect and fear or expectancy}

On average, pain expectancy was greater in the pain-associated context [mean $(\mathrm{SD})=7.5$ (3.0)] compared with the vibration-associated environment [1.9 (2.4)]. Similarly, greater fear was reported in the pain-associated environment [mean (SD) fear rating $=4.0(2.4)]$ than in the vibrotactileassociated environment [mean $(\mathrm{SD})$ fear rating $=1.6(1.7)]$. The difference in fear between environments appeared to have a weak but significant relationship with difference in pain $(r=0.39, p=0.03)$. This was not true for expectancy ratings $(r=0.25, p=0.17)$. 


\section{Discussion}

The hypothesis that pain-associated cues can increase or even provoke pain has gained attention (Madden et al. 2016; Moseley and Vlaeyen 2015). Studies have confirmed that pain-associated cues can enhance pain, but not to a degree that would be considered meaningful in clinical scenarios. Consistent with our previous research, we found that an effect of pain-associated VR environmental contexts was present only under certain conditions (Harvie et al. 2018). That is, an effect of environment was detected only when the reported pain during the learning phase was sufficiently intense. The effect of VR environment was also dependent on the intensity of the painful test stimulus it was paired with-we only found an effect on the higher intensity stimulus.

\section{Pain as a driver of learning}

In classical fear conditioning studies, the more painful the unconditioned stimulus, the fewer trials it takes to establish an aversive emotional association with a previously neutral stimulus (Schafe et al. 2001; Apkarian 2008). Our findings support the suggestion that classically conditioned hyperalgesia also depends on pain intensity during learning, in those only participants meeting our a priori cut-off for sufficient pain intensity showed any effect.

\section{The role of test stimulus intensity}

Based on perceptual models, we hypothesised that painassociated contexts would have their strongest pain-enhancing effect on painful stimuli of low intensity. Instead, statistically significant modulation in the pain-associated context relative to the vibration-associated context was seen only when the test stimulus was more intense (mean difference: $11.4(25.7) \%$ ). The reason why the relationship may be in the opposite to expected direction is difficult to discern from the current data.

\section{The relationship between fear, expectancy, and pain}

It has been suggested that pain-related fear or expectancy may be responsible for classically conditioned hyperalgesia. In our data, only $15 \%$ of the variance in pain ratings was explained by fear $\left(r^{2}=0.15\right)$, while expectancy explained just $6 \%\left(r^{2}=0.06\right)$. While the current design precludes inferences of causation, our findings are remarkably consistent with mediation analyses that show that a $14 \%$ change in pain can be explained by fear (Meulders et al. 2012).

\section{Limitations}

The major limitation of this study was that, although we calibrated our highest intensity to $7 / 10$, the average reported intensity for that stimulus during conditioning was only 5.4 (1.2)/10. Since high-intensity stimuli may be important to achieving an effect, our study may be limited by the modest intensity of pain reported by participants, even after removal of those reporting the least amount of pain. The need to remove participants not reporting sufficient pain resulted in the further limitation of low participant numbers, which limits confidence in our findings. Among other limitations, participants were not drawn from a patient or pain-susceptible population, although evidence suggests that some individuals may be more susceptible to aberrant pain-related classical conditioning (Harvie et al. 2017).

\section{Clinical relevance of conditioned hyperalgesia}

The belief that pain may be a classically conditioned response is a widespread clinical belief (Madden and Moseley 2016). In clinical scenarios, changes in pain of less than $30 \%$ are considered non-meaningful. As such, our study is consistent with the broader literature which has so far failed to prove that clinically meaningful changes in pain can be induced through classical conditioning. Nonetheless, the small effects seen in laboratory studies such as our study may be less than what is possible in clinical scenarios.

\section{Conclusion}

While the effect was small, the results are consistent with the proposition that pain-associated cues may induce hyperalgesia to some degree, under certain conditions. In particular, results highlight the potential relevance of stimulus intensity during and after the initial painful experience. Further attention to understanding the variables that impact classically conditioned hyperalgesia may contribute to determining its potential relevance.

Supplementary Information The online version contains supplementary material available at https://doi.org/10.1007/s00221-021-06285-4.

Acknowledgements The authors would like to thank Associate Professor Ross Smith (Wearable Computer Lab, University of South Australia) for creating the Virtual Reality software that enabled simple display of, and interchange between, the scenes.

Author contributions All authors contributed meaningfully to the design, analysis interpretation, and manuscript preparation.

Funding DH is supported by an Early Career Research Fellowship from the National Health and Medical Research Council of Australia 
(GNT1142929). VJM is supported by the Fogarty International Center of the National Institutes of Health (award K43TW011442). The content is solely the responsibility of the authors and does not necessarily represent the official views of the National Institutes of Health.

Availability of data and material Data have been made publicly available through Harvard's Dataverse: https://doi.org/10.7910/DVN/ MFD7JW.

\section{Code availability N/A.}

\section{Declarations}

Conflict of interest DH, MWC and VJM receive speakers' fees for talks on pain and rehabilitation.

Ethics approval All procedures conformed to the Declaration of Helsinki and were approved by Griffith University Human Research Ethics (GU Ref No: 2016/242).

Consent to participant All participants gave written informed consent.

Consent to publish All participants gave written consent for the publication of de-identified data.

Open Access This article is licensed under a Creative Commons Attribution 4.0 International License, which permits use, sharing, adaptation, distribution and reproduction in any medium or format, as long as you give appropriate credit to the original author(s) and the source, provide a link to the Creative Commons licence, and indicate if changes were made. The images or other third party material in this article are included in the article's Creative Commons licence, unless indicated otherwise in a credit line to the material. If material is not included in the article's Creative Commons licence and your intended use is not permitted by statutory regulation or exceeds the permitted use, you will need to obtain permission directly from the copyright holder. To view a copy of this licence, visit http://creativecommons.org/licenses/by/4.0/.

\section{References}

Apkarian AV (2008) Pain perception in relation to emotional learning. Curr Opin Neurobiol 18:464-468. https://doi.org/10.1016/j.conb. 2008.09.012

Domjan M, Galef BG (1983) Biological constraints on instrumental and classical conditioning: retrospect and prospect. Anim Learn Behav 11:151-161

Farrar JT, Young JP Jr, LaMoreaux L, Werth JL, Poole MR (2001) Clinical importance of changes in chronic pain intensity measured on an 11-point numerical pain rating scale. Pain 94:149-158. https://doi.org/10.1016/S0304-3959(01)00349-9

Glotzbach E, Ewald H, Andreatta M, Pauli P, Muhlberger A (2012) Contextual fear conditioning predicts subsequent avoidance behaviour in a virtual reality environment. Cogn Emot 26:12561272. https://doi.org/10.1080/02699931.2012.656581

Hamm AO, Vaitl D, Lang PJ (1989) Fear conditioning, meaning, and belongingness: a selective association analysis. J Abnorm Psychol 98:395-406. https://doi.org/10.1037//0021-843x.98.4.395

Harvie DS, Meulders A, Madden VJ, Hillier SL, Peto DK, Brinkworth R, Moseley GL (2016) When touch predicts pain: predictive tactile cues modulate perceived intensity of painful stimulation independent of expectancy. Scand J Pain 11:11-18
Harvie DS, Moseley GL, Hillier SL, Meulders A (2017) Classical conditioning differences associated with chronic pain: a systematic review. J Pain 18:889-898. https://doi.org/10.1016/j.jpain.2017. 02.430

Harvie DS, Sterling M, Smith AD (2018) Do pain-associated contexts increase pain sensitivity? An investigation using virtual reality. Scand J Pain 18:525-532

Jensen KB, Kaptchuk TJ, Kirsch I et al (2012) Nonconscious activation of placebo and nocebo pain responses. Proc Natl Acad Sci USA 109:15959-15964. https://doi.org/10.1073/pnas.1202056109

Jensen K, Kirsch I, Odmalm S, Kaptchuk TJ, Ingvar M (2015) Classical conditioning of analgesic and hyperalgesic pain responses without conscious awareness. Proc Natl Acad Sci 112:7863-7867

Knill DC, Pouget A (2004) The Bayesian brain: the role of uncertainty in neural coding and computation. Trends Neurosci 27:712-719. https://doi.org/10.1016/j.tins.2004.10.007

Kroenke K, Spitzer RL (2002) The PHQ-9: a new depression diagnostic and severity measure. Psychiatr Ann 32(9):509-515

Lethem J, Slade PD, Troup JD, Bentley G (1983) Outline of a fearavoidance model of exaggerated pain perception-I. Behav Res Ther 21:401-408. https://doi.org/10.1016/0005-7967(83)90009-8

Liu CC, Crone NE, Franaszczuk PJ, Cheng DT, Schretlen DS, Lenz FA (2011) Fear conditioning is associated with dynamic directed functional interactions between and within the human amygdala, hippocampus, and frontal lobe. Neuroscience 189:359-369

Lovibond PF, Shanks DR (2002) The role of awareness in Pavlovian conditioning: empirical evidence and theoretical implications. J Exp Psychol Anim Behav Process 28:3-26

Lowe B, Decker O, Muller S, Brahler E, Schellberg D, Herzog W, Herzberg PY (2008) Validation and standardization of the Generalized Anxiety Disorder Screener (GAD-7) in the general population. Med Care 46:266-274. https://doi.org/10.1097/MLR.0b013 e318160d093

Madden VJ, Moseley GL (2016) Do clinicians think that pain can be a classically conditioned response to a non-noxious stimulus? Man Ther 22:165-173

Madden VJ, Harvie DS, Parker R, Jensen KB, Vlaeyen JW, Moseley GL, Stanton TR (2016) Can pain or hyperalgesia be a classically conditioned response in humans? A systematic review and metaanalysis. Pain Med 17(6):1094-1111

McNeil DW, Kennedy SG, Randall CL, Addicks SH, Wright CD, Hursey KG, Vaglienti R (2018) Fear of Pain Questionnaire-9: brief assessment of pain-related fear and anxiety. Eur J Pain 22:39-48. https://doi.org/10.1002/ejp.1074

Meulders A, Vansteenwegen D, Vlaeyen JWS (2011) The acquisition of fear of movement-related pain and associative learning: a novel pain-relevant human fear conditioning paradigm. Pain 152:2460 2469. https://doi.org/10.1016/j.pain.2011.05.015

Meulders A, Vansteenwegen D, Vlaeyen JW (2012) Women, but not men, report increasingly more pain during repeated (un) predictable painful electrocutaneous stimulation: evidence for mediation by fear of pain. PAIN ${ }^{\circledR} 153: 1030-1041$

Meyniel F, Sigman M, Mainen ZF (2015) Confidence as Bayesian probability: from neural origins to behavior. Neuron 88:78-92

Mitchell CJ, De Houwer J, Lovibond PF (2009) The propositional nature of human associative learning. Behav Brain Sci 32:183198. https://doi.org/10.1017/S0140525X09000855 (discussion 198-246)

Moseley GL, Vlaeyen JWS (2015) Beyond nociception: the imprecision hypothesis of chronic pain. Pain 156:35-38. https://doi.org/ 10.1016/j.pain.0000000000000014

Pavlov IP (1928) Lectures on conditioned reflexes: twenty-five years of objective study of the higher nervous activity (behaviour) of animals. (Translated by: Gantt WH). Liverwright Publishing Corporation, New York. https://doi.org/10.1037/11081-000 
Schafe GE, Nader K, Blair HT, LeDoux JE (2001) Memory consolidation of Pavlovian fear conditioning: a cellular and molecular perspective. Trends Neurosci 24:540-546. https://doi.org/10.1016/ s0166-2236(00)01969-x

Smith A, Carlow K, Biddulph T, Murray B, Paton M, Harvie DS (2017) Contextual modulation of pain sensitivity utilising virtual environments. Br J Pain 11:71-80. https://doi.org/10.1177/2049463717 698349

Sullivan MJ, Bishop SR, Pivik J (1995) The pain catastrophizing scale: development and validation. Psychol Assess 7:524

Van Damme S, Lorenz J, Eccleston C, Koster EH, De Clercq A, Crombez G (2004) Fear-conditioned cues of impending pain facilitate attentional engagement. Neurophysiol Clin 34:33-39. https://doi.org/10.1016/j.neucli.2003.11.001

Vlaeyen JWS, Linton SJ (2000) Fear-avoidance and its consequences in chronic musculoskeletal pain: a state of the art. Pain 85:317-332. https://doi.org/10.1016/S0304-3959(99)00242-0
Vlaeyen JWS, Linton SJ (2012) Fear-avoidance model of chronic musculoskeletal pain: 12 years on. Pain 153:1144-1147. https://doi. org/10.1016/j.pain.2011.12.009

Vlaeyen JW, Kole-Snijders AM, Rotteveel AM, Ruesink R, Heuts PH (1995) The role of fear of movement/(re)injury in pain disability. J Occup Rehabil 5:235-252

Publisher's Note Springer Nature remains neutral with regard to jurisdictional claims in published maps and institutional affiliations. 